\title{
A COMPUTER BASED CATALOGUE OF OPEN CLUSTER DATA
}

Gösta Lyngà and Ingemar Lundström Lund Observatory

The catalogue contains data for 1130 galactic clusters. Each cluster has 255 available storage places. Most of these are reserved for positional, descriptional and physical parameters. In the present version the following items are included:

- Cluster name, OC1 No. (A1ter et al. 1970)

- R.A., Dec (1950.0), R.A., Dec. (2000.0), precession for 10 years, galactic longitude, galactic latitude

- Data on peculiar stars (Feinstein 1979): Type, number, references

- Part of Dixon's (1976) catalogue: POSS chart type, chart No., coordinates for chart centre (R.A., Dec.), position on chart, largest published angular diameter, magnitude of brightest star, Trumpler class, nebulosity

- Part of Bochum-Strasbourg catalogue (Kluke et al. 1975): Selected angular diameter, distance, magnitude of brightest star, spectral class, total magnitude, colour excess Buscombe's (1978) compilation: Radial velocity, distance, colour excess, total apparent magnitude, total absolute magnitude, linear diameter, age, mass

- Basel data (Becker and Fenkart 1971; Fenkart and Binggeli 1979): Photometric system, colour excess, interstellar extinction, distance, spectral type of hottest star, colour type of bluest star, linear diameter, type of observation

- van den Bergh-Hagen (1975) data: Angular diameter, richness

Lyngå (1979) compilation: Selected angular diameter, references (various other parameters with references will be included later). 
The last 18 storage places are reserved for tracers, which show the order of objects according to a given parameter by pointing to the next object (positive tracer) or the preceding one (negative tracer). Thus, e.g. by following the numbers in storage place 249 we will obtain a listing in order of R.A. $(1950.0)$, while the numbers in storage place 251 give us the catalogue arranged in galactic longitude. This system of addressing makes the catalogue more flexible than one particular sequential ordering would have done. Adding or excluding an entry is easily managed by the altering of tracers.

The catalogue is available as magnetic type, microfiche or listing through the Stellar Data Centre in Strasbourg. It will continuously be updated and thus no printed version is foreseen.

The catalogue also includes a detailed description including complete references and an alibi list for cluster names.

\section{REFERENCES}

Alter, G., Balázs, B., and Ruprecht, J.: 1970, Catalogue of Star Clusters and Associations (2nd Ed.), Budapest.

Becker, W., and Fenkart, R.: 1971, Astron. Astrophys. Suppl. 4, 241 .

Buscombe, W.: 1978, A compilation of open cluster parameters

(private communication).

Dixon, R.S.: 1976, Proc. IAU Col1. No. 35, 167.

Feinstein, A.: 1979, A list of peculiar stars in open clusters (private communication).

Fenkart, R., and Binggeli, B.: 1979, Astron. Astrophys. Supp Z. 35, 271 .

Kluke, J., Moffat, A.F.J., Schmidt-Kaler, Th., and Vogt, N.: 1975, Excerpt from the catalogue of star clusters and associations by Alter et al., available from CDS, Strasbourg.

Lyngå, G.: 1979 (in preparation) .

van den Bergh, S., Hagen, G.L.: 1975, Astron. J. 80, 11. 